Check for updates

Cite this: Phys. Chem. Chem. Phys., 2019, 21, 2186

Received 15th November 2018, Accepted 8th January 2019

DOI: $10.1039 / c 8 c p 07077 f$

rsc.li/pccp

\title{
Thermodynamic limits of countercurrent reactor systems, with examples in membrane reactors and the ceria redox cycle
}

\begin{abstract}
Brendan Bulfin (D)
Countercurrent reactors can be utilized in chemical reaction systems which involve either a reaction between flows of different phases, or reactions between flows separated by a selective permeable membrane. This idea is quite similar in nature to a countercurrent heat exchanger, where the inlet of one participating flow is exposed to the outlet of the opposite flow. A countercurrent configuration can therefore improve the reaction conversion extent and transport properties. Here we formulate a straightforward approach in terms of an exchange coordinate, in order to determine an upper bound of species exchange in such systems, subject to the second law of thermodynamics and conservation of mass. The methodology is independent of the specifics of reactor design and can be generally applied to determine the maximum thermodynamic benefit of using a countercurrent reactor. We then demonstrate the analysis for a number of thermochemical fuel production routes; membrane thermolysis of carbon dioxide, dry methane reforming across a membrane, reverse water gas shift across a membrane, and the thermochemical ceria cycle.
\end{abstract}

\section{Introduction}

Countercurrent exchange systems are widely applied in industry and frequently observed in nature. For example, a heat exchanger can be arranged in a countercurrent configuration in order to improve overall heat transfer. The same concept is also useful to improve chemical species transfer from one flow to another. A simple example of this occurring in nature is that of gills in fish, which utilize a countercurrent flow arrangement of water and blood to achieve favourable transfer of oxygen. ${ }^{1}$

In chemical processes with two distinct reacting streams which exchange a species, say A, it may be beneficial to use a countercurrent configuration. This is possible if the reactants have different phases, such as a stream of solid particles reacting with a flow of gas, or bubbles of gas rising against a liquid current. ${ }^{2}$ It can also be applied to flows of the same phase if they are separated by an interface such as a species selective membrane, ${ }^{3}$ as illustrated in Fig. 1 . Despite the common application of such systems, the author couldn't find a standard methodology to determine the thermodynamic limits of countercurrent reactors, either in text books or the literature. A number of models have been developed and applied to specific cases, ${ }^{4-6}$ but there is a need for a more generalised approach.

Department of Mechanical and Process Engineering, ETH Zürich, 8092 Zürich, Switzerland. E-mail: bulfinb@ethz.ch

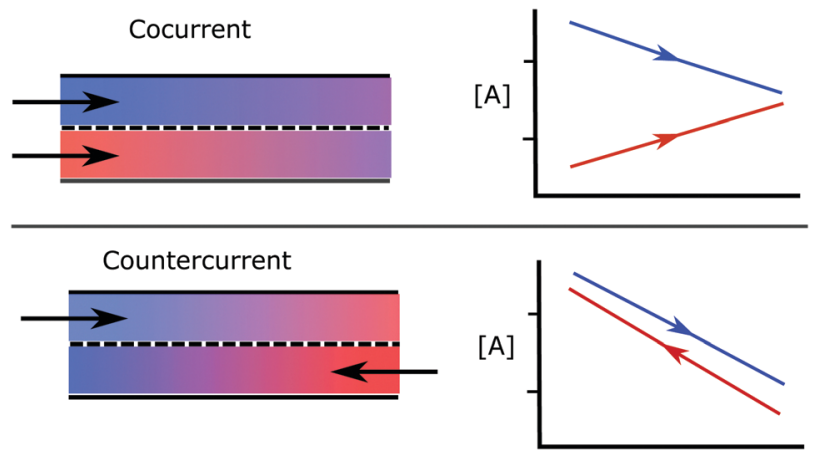

Fig. 1 A schematic comparing countercurrent and cocurrent flow, where a species $A$ is exchanged between the two flows.

The lack of such a standard methodology has lead many authors to apply simplified models, leading to unphysical results. These errors are prevalent in the field of thermochemical fuel production via either membrane reactors or redox cycles. ${ }^{7-12}$ Thermochemical fuel production systems are proposed as a means of converting heat to chemical energy, by driving chemical reactions that produce a fuel such as syngas. Many authors take the approach of setting the concentration [A] at the exit of each flow to be equal to the concentration at the inlet of the opposite incoming stream. In Fig. 1, this would mean the two lines meet at both ends, which is appealing for it's simplicity. However, this ignores the capacity of each flow to take 
up or release the species which is exchanged. Applying such a model can violate both the second law of thermodynamics and conservation of mass. An analogous error in countercurrent heat exchangers would be to assume that the temperature can be matched at both ends, regardless of the relative flow rates or heat capacities of the participating flows.

This work aims to give researchers a straightforward approach to determine an upper bound on the amount of species exchanged in countercurrent reacting flows. A simple methodology based on a species exchange is developed and used to analyse several examples in thermochemical fuel production systems. The methodology is applied both analytically and numerically where an algorithm is outlined for use with thermodynamic software, with links to my implementation made public on GitHub. $\dagger$ The methodology is also developed in a general way and broader in context than the examples discussed.

\section{Thermodynamic methodology}

Consider two distinct streams of matter, which can exchange a species A from one flow to another (flow 1 to flow 2), as illustrated in Fig. 1. For the sake of determining upper bounds we would like to consider a very idealized case, which does not consider any irreversible effects, such as diffusion along the flows. The system is therefore simplified with the following assumptions.

- The system is considered to be operating in a steady state, with temperature, pressure, flow rates, species concentration profiles, and heat consumption all assumed to be constant in time.

- Both streams are considered to be in plug flow with no diffusion along the flow direction, and perfectly mixed perpendicular to the flow.

With these assumptions the exchanger can then be considered as a one dimensional interface of length $l$, along which the species A can be exchanged, as illustrated in Fig. 2. In order to have a spontaneous process with the transfer of species $\mathrm{A}$ from flow 1 to flow 2, we must have,

$$
\mu_{\mathrm{A}, 1}(x) \geq \mu_{\mathrm{A}, 2}(x), \forall x \in[0, l],
$$

where $\mu_{\mathrm{A}, 1}$ and $\mu_{\mathrm{A}, 2}$ are the chemical potentials of species $\mathrm{A}$ in flow 1 and 2 respectively.

It is more convenient to formulated the problem to be independent of the exchanger size and the position coordinates. This is achieved here by defining an exchange coordinate $\kappa$, as the number of moles of species A that have been exchanged per mole of flow 1 entering the system, by a certain point $x$ along the interface, which is given by

$$
\kappa(x)=\frac{\int_{0}^{x}\left|j_{\mathrm{A}}(x)\right| \mathrm{d} x}{\dot{n}_{1}},
$$

where $\dot{n}_{1}\left[\mathrm{~mol} \mathrm{~s}^{-1}\right]$ is the molar flow rate of flow 1 , and $j_{\mathrm{A}}(x)\left[\mathrm{mol} \mathrm{m} \mathrm{m}^{-1} \mathrm{~s}^{-1}\right]$ is the molar flux of species A from flow 1 to flow 2 as a function of the position, $x$. Since there is an

$\dagger$ https://github.com/bulfinb/countercurrent_reactor_algorithm. integral over the length in the numerator, this gives a dimensionless exchange coordinate. For simple systems $\kappa$ will be a monotonic function of $x$, and the change of co-ordinates is trivial. Eqn (2) then only serves as a formal definition and the system is simply analysed with respect to the exchange coordinate $\kappa$. This gives a much more convenient analysis, as $\kappa$ corresponds directly to changing species number in the flows, and can easily be related to the equilibrium thermodynamics of both flows. We are now interested in finding $\mu_{\mathrm{A}}(\kappa)$ for $\kappa \in\left[0, \kappa_{\text {total }}\right]$, in both flows, where $\kappa_{\text {total }}=\kappa_{1}(l)$ is the exchange parameter at the exit of the flow (i.e. the total species exchange within the system).

With this change of coordinates eqn (1) becomes,

$$
\mu_{\mathrm{A}, 1}(\kappa) \geq \mu_{\mathrm{A}, 2}(\kappa) . \forall \kappa \in\left[0, \kappa_{\text {total }}\right] .
$$

In words, for any species exchange coordinate $\kappa$, the chemical potential of the species A in flow 1, must be greater than or equal to that in flow 2 .

The conservation of mass can be applied to the exchange between the flows, meaning that the number of moles of A to have left flow 1 , must be equal to the number of moles to have entered flow 2, at all points along the reactor interface. In a cocurrent system this statement is mathematically trivial and simply means that

$$
\kappa_{1}=\kappa_{2} \equiv \kappa,
$$

where $\kappa_{i}$ is the exchange coordinate defined with the integral starting at the inlet of each flow $i$. For both streams this is equivalent to our definition of the exchange coordinate above $\kappa$.

In a countercurrent system we have flow 2 reversed and so conservation of mass means that the exchange coordinate $\kappa$ can be redefined in flow 2 by,

$$
\kappa_{1}=\kappa_{2}-\kappa_{\text {total }} \equiv \kappa,
$$

where $\kappa_{2}$ is calculated by changing limits of the integral in eqn (2) to be from $l$ to $x$. This is a simple transformation, where $\kappa_{2}$ would be 0 when $\kappa_{1}=\kappa_{\text {total }}$ and vice versa as seen in Fig. 2 . Essentially, this reverses flow 2's dependence on the exchange coordinate $\kappa$, relative to the cocurrent case, which is illustrated in Fig. 3.

For the cocurrent case, the chemical potential $\mu_{\mathrm{A}, 1}$ is expected to be a decreasing function of $\kappa$, and $\mu_{\mathrm{A}, 2}$ an increasing function,

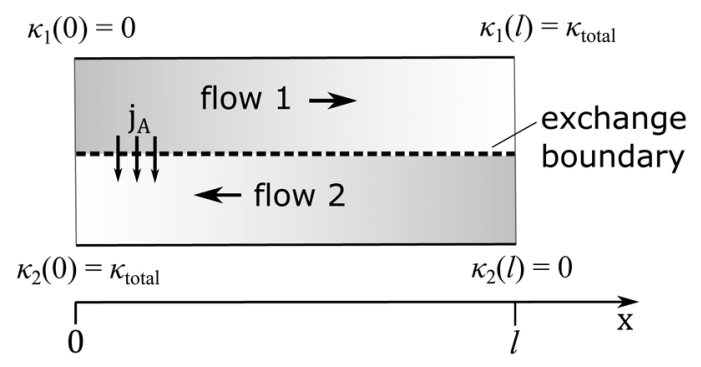

Fig. 2 A schematic showing two flows in countercurrent configuration and the difference in exchange coordinate defined with respect to either flow. The flows are separated by an exchange boundary, which could be a phase boundary or a species selective membrane. 

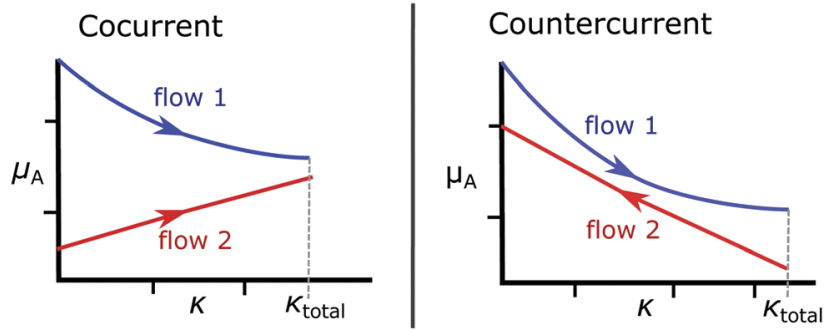

Fig. 3 A schematic showing the advantage of countercurrent flow over cocurrent flow, where the countercurrent case allows for a greater exchange of species.

which is illustrated in Fig. 3. This behaviour ensures thermodynamic stability, where any addition of species A to a solution should not decrease $\mu_{\mathrm{A}}$, and vice versa. Therefore, in a cocurrent system, it is sufficient to obey eqn (3) at the end point of the system $\kappa=\kappa_{\text {total }}$, and the thermodynamic upper bound for species exchange $\kappa_{\text {total }}=\kappa_{\max }$ would be the case where they are equal at the outlet,

$$
\mu_{\mathrm{A}, 1}\left(\kappa_{\text {total }}\right)=\mu_{\mathrm{A}, 2}\left(\kappa_{\text {total }}\right) .
$$

A countercurrent system is not so straightforward. Since flow 2's dependence on $\kappa$ is reversed (eqn (5)), the chemical potentials $\mu_{\mathrm{A}, 1}$ and $\mu_{\mathrm{A}, 2}$ will both be decreasing functions of $\kappa$ as illustrated in Fig. 3. This means that with non-linear dependence on $\kappa$, it may not be possible to have equal concentrations at ether of the end points, without violating eqn (3) somewhere in the domain $\kappa \in\left(0, \kappa_{\text {total }}\right)$.

For smooth functions this implies that they could meet at one of the boundaries,

$$
\mu_{\mathrm{A}, 1}(0)=\mu_{\mathrm{A}, 2}(0) \text { or } \mu_{\mathrm{A}, 1}\left(\kappa_{\text {total }}\right)=\mu_{\mathrm{A}, 2}\left(\kappa_{\text {total }}\right),
$$

that they share a common tangent somewhere in between, $\kappa \in$ $\left(0, \kappa_{\text {total }}\right)$, which would satisfy

$$
\frac{\partial \mu_{\mathrm{A}, 1}}{\partial \kappa}=\frac{\partial \mu_{\mathrm{A}, 2}}{\partial \kappa} \text { and } \mu_{\mathrm{A}, 1}(\kappa)=\mu_{\mathrm{A}, 2}(\kappa) .
$$

or, it is also possible that all of species A is transferred from flow 1 to flow 2, eqn (3) holds, but neither of the conditions in eqn (7) and (8) are satisfied. In countercurrent reactors it is therefore important to carefully consider the reaction systems of interest.

To determine the upper bound of species exchange for a given system one must first fix some parameters such as the temperatures and pressures of the streams. Another key physical parameter which can be set is the relative molar flow rates, which is denoted in this work by $\omega$,

$$
\omega=\frac{\dot{n}_{2}}{\dot{n}_{1}} .
$$

For each flow one should then formulate a suitable state function,

$$
\begin{aligned}
& f_{1}\left(T_{1}, p_{1}, \mu_{\mathrm{A}, 1}, \kappa\right)=0, \\
& f_{2}\left(T_{2}, p_{2}, \mu_{\mathrm{A}, 2}, \kappa\right)=0,
\end{aligned}
$$

which can be used to determine the relation between the chemical potentials and the exchange coordinate $\mu_{\mathrm{A}, i}(\kappa)$. With the assumption here of plug flow with no diffusion along the flow's and perfect mixing perpendicular, the Gibbs free energy gives such a suitable state function.

A simple method of determining the thermodynamic limit on species exchange is then to start with $\kappa_{\text {total }}=0$ and increase this value, until one of the limiting conditions are reached (6 for cocurrent systems, and 7 and 8 for countercurrent), or as is possible for countercurrent systems, all of the species is transferred.

If the flow's are in contact or separated by a thin membrane, most cases will have $T_{1}=T_{2}$ and $p_{1}=p_{2}$, but the methodology is by no means limited to these cases. For example one could conceivably have a pressure or temperature difference across a membrane, and as long as eqn (3) holds, then we can have spontaneous process with the transfer of species from flow 1 to flow 2 . The temperature and pressure could also vary within the system. For example in an adiabatic reactor, where the temperature could also depend on the exchange coordinate $T(\kappa)$ as a result of the heat of the reaction. The examples here are heat driven reactors, which are best approximated as isothermal rather than adiabatic, and so we set both temperatures and pressures to be equal and constant in both flows $T_{1}=T_{2}=T$ and $p_{1}=p_{2}=p$.

It is important to also understand the context in which the model can be applied. Real countercurrent reactors are open systems which can have irreversible effects, such as diffusion along the flow's, and a more sophisticated model would be required to accurately predict performance. With the assumptions used here one can simply set an upper bound on species exchange. It therefore serves as a straightforward check of thermodynamic limits and a means of determining the potential for performance improvement over a cocurrent system. It should also be noted that changes in interface energy have been omitted from the analysis, which in some cases, such as bubble reactors, may play an important role.

\section{Examples}

This section illustrates the analysis of a number of countercurrent reactor systems and makes a brief comparison to previous models and experimental data available in the literature. The examples we consider are,

- thermolysis of $\mathrm{CO}_{2}$ with oxygen removal across a membrane,

- dry reforming with oxygen exchange across a membrane,

- reverse water gas shift with oxygen exchange across a membrane,

- $\mathrm{CeO}_{2}$ reduction with a sweep gas removing oxygen,

which are illustrated in Fig. 4.

Cases (a) and (d) are solved analytically. Cases (b) and (c) are treated with a more robust numerical method utilizing the thermodynamic library Cantera, ${ }^{13}$ with a simple implementation of the methodology in python. $\dagger$ 


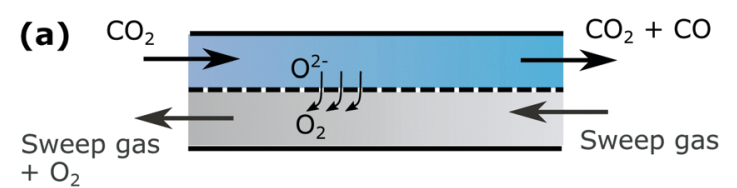

(c)

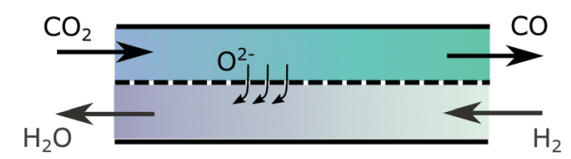

(b)

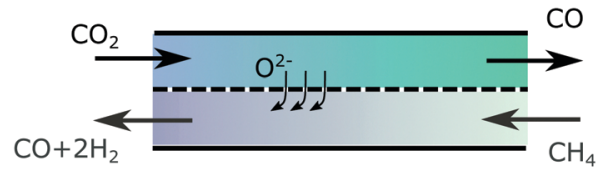

(d)

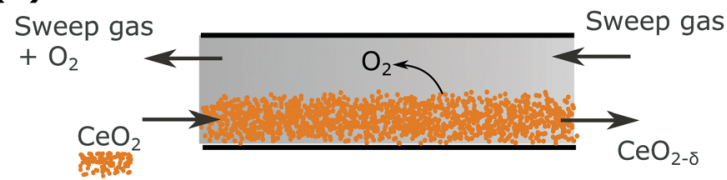

Fig. 4 A schematic showing the examples $(a-d)$ in countercurrent configuration.

\section{(a) Membrane thermolysis}

The idea of using a species selective membrane to separate the products of steam thermolysis has been proposed by Fletcher et al. as early as $1977,{ }^{14,15}$ with the reaction given by,

$$
\mathrm{H}_{2} \mathrm{O} \stackrel{\text { heat }}{\longrightarrow} \mathrm{H}_{2}+\frac{1}{2} \mathrm{O}_{2} \text {. }
$$

It is suggested that concentrated solar power could supply the heat for this reaction, and one or both of the products can be selectively removed from the steam using a membrane selective to either hydrogen or oxygen.

This direct thermolysis method has been experimentally demonstrated by Tou et al., using a concentrated solar powered reactor to split $\mathrm{CO}_{2}$ with an oxygen selective membrane made from ceria. ${ }^{12}$ In this system argon and carbon dioxide were arranged in countercurrent flow on either side of a ceria membrane, with the argon acting as an inert sweep gas to carry away oxygen produced by the thermolysis reaction,

$$
\mathrm{CO}_{2} \stackrel{\text { heat }}{\longrightarrow} \mathrm{CO}+\frac{1}{2} \mathrm{O}_{2} \text {. }
$$

The heat was supplied using a solar simulator, and the net result was the transfer of oxygen out of the $\mathrm{CO}_{2}$ flow, producing excess CO. Thermodynamic analysis of this countercurrent system utilized a simplified approach of matching the oxygen partial pressure at both inlets and outlets of the reactor, ${ }^{11,12}$ which gives unphysical overoptimistic results. Li et al. have provided a more physical model of this system based on a Gibbs critereon $\mathrm{d} G_{T, p} \leq 0,{ }^{30}$ which is in agreement with the results presented here.

A schematic of the system can be seen in Fig. 4(a), which can be modeled according to the methodology described in the previous section to determine the thermodynamic limits. Note that the analysis presented would be identical if the $\mathrm{CO}_{2}$ were replaced with steam for $\mathrm{H}_{2} \mathrm{O}$ thermolysis.

The system is modeled as isothermal at a temperature of $1500{ }^{\circ} \mathrm{C}$, and with both flows at a pressure of 1 bar, which allows for direct comparison of our model to the experimental work of Tou et al. ${ }^{12}$ Since oxygen is being exchanged between the two flows, we can define our exchange coordinate as

$$
\kappa=\frac{\int_{0}^{l}\left|j_{\mathrm{O}_{2}}(x)\right| \mathrm{d} x}{\dot{n}_{\mathrm{CO}_{2}}},
$$

In this case $\kappa=0.5$ would correspond to complete transfer of the oxygen and a pure stream of CO leaving the reactor.

The relative flow rate of the sweep gas to the $\mathrm{CO}_{2}$ is used as a free control parameter,

$$
\omega=\frac{\dot{n}_{\text {sweep }}}{\dot{n}_{\mathrm{CO}_{2}}} .
$$

The thermodynamics of both flows can be well approximated as ideal gas solutions giving

$$
\mu_{\mathrm{O}_{2}}=\mu_{\mathrm{O}_{2}}^{\circ}+R T \ln \left(\frac{p_{\mathrm{O}_{2}}}{p^{\circ}}\right) .
$$

Both flows have equal temperature, and so we can use the oxygen partial pressure instead of the chemical potential in eqn (3) giving the condition,

$$
p_{\mathrm{O}_{2}, 1}(\kappa) \geq p_{\mathrm{O}_{2}, 2}(\kappa) . \forall \kappa \in\left[0, \kappa_{\text {total }}\right]
$$

We can also use the partial pressure to check the conditions given in eqn (6)-(8). We need to determine $p_{\mathrm{O}_{2}}(\kappa)$ for both streams. Once we have these functions we can apply the methodology developed and determine the maximum exchange extent $\kappa_{\max }$ for a given temperature pressure and relative flow rates.

For the sweep gas determining $p_{\mathrm{O}_{2}}(\kappa)$ is straightforward. Assuming we have a sweep gas with an oxygen impurity $\phi=\frac{n_{\mathrm{O}_{2}}}{n_{\text {sweep }}}$, flowing into the system, the partial pressure of oxygen in the sweep gas stream (flow 2) is given by

$$
p_{\mathrm{O}_{2}, 2}(\kappa)=\frac{\phi \omega+\kappa}{\omega+\kappa} p .
$$

The derivative of this function is then given by,

$$
\frac{\partial p_{\mathrm{O}_{2}, 2}}{\partial \kappa}=\frac{\omega-\phi \omega}{(\omega+\kappa)^{2}} p
$$

In the countercurrent case we use the substitution $\kappa^{\prime}=\kappa_{\text {total }}-\kappa$ in eqn (18) and (19).

In the case of $\mathrm{CO}_{2}$, we must consider the equilibrium thermodynamics of the reaction given in eqn (13). The equilibrium 
composition of the $\mathrm{CO}_{2}$ splitting reaction is described by the variance in the Gibbs free energy,

$$
\Delta G(T)=\Delta G^{\circ}(T)+R T \ln \left(\frac{p_{\mathrm{CO}}\left(\frac{p_{\mathrm{O}_{2}}}{p^{\circ}}\right)^{0.5}}{p_{\mathrm{CO}_{2}}}\right)=0
$$

which should be zero at equilibrium. This assumes the formation of other species (e.g. atomic oxygen) is negligible and the partial pressures are related by,

$$
p_{\mathrm{CO}}+p_{\mathrm{CO}_{2}}+p_{\mathrm{O}_{2}}=1 \text {. }
$$

Eqn (20) and (21) can be solved to get $p_{\mathrm{CO}}$ and $p_{\mathrm{CO}_{2}}$ for a given $p_{\mathrm{O}_{2}}$,

$$
\begin{gathered}
p_{\mathrm{CO}}\left(p_{\mathrm{O}_{2}}\right)=\frac{\left(p-p_{\mathrm{O}_{2}}\right) K}{\left(\frac{p_{\mathrm{O}_{2}}}{p^{\circ}}\right)^{0.5}+K} \\
p_{\mathrm{CO}_{2}}\left(p_{\mathrm{O}_{2}}\right)=p-p_{\mathrm{CO}}\left(p_{\mathrm{O}_{2}}\right)-p_{\mathrm{O}_{2}},
\end{gathered}
$$

where $K=\exp \left(\frac{-\Delta G^{\circ}}{R T}\right)$ is the equilibrium constant.

The exchange coordinate in the $\mathrm{CO}_{2}$ flow can be formulated in terms of the partial pressures as,

$$
\kappa=\frac{0.5 p_{\mathrm{CO}}}{p_{\mathrm{CO}}+p_{\mathrm{CO}_{2}}}-\frac{p_{\mathrm{O}_{2}}}{p_{\mathrm{CO}}+p_{\mathrm{CO}_{2}}},
$$

where the first term is the total extent of oxygen formation in the splitting reaction, and the second term accounts for the oxygen gas which remains in flow 1. Subbing eqn (22) and (23) into eqn (24) gives

$$
\kappa\left(p_{\mathrm{O}_{2}}\right)=\frac{0.5 K^{\circ}}{K^{\circ}+\left(\frac{p_{\mathrm{O}_{2}}}{p^{\circ}}\right)^{0.5}}-\frac{p_{\mathrm{O}_{2}}}{p-p_{\mathrm{O}_{2}}},
$$

which can then be inverted to get $p_{\mathrm{O}_{2}, 1}(\kappa)$, although this does not yield a simple analytical expression. For that reason numerical solutions of eqn (25) for a given $\kappa$ were used. For the derivative we can use the calculus identity

$$
\frac{\partial p_{\mathrm{O}_{2}}}{\partial \kappa}=\frac{1}{\frac{\partial \kappa}{\partial p_{\mathrm{O}_{2}}}}
$$

to determine $\frac{\partial p_{\mathrm{O}_{2}, 1}}{\partial \kappa}$.

The thermodynamic limiting case can then be found, by starting from $\kappa_{\text {total }}=0$, and increasing this value until our stop conditions given in eqn (6)-(8) are reached, corresponding to $\kappa_{\text {total }}=\kappa_{\max }$. An example of the limiting case is illustrated in Fig. 5(a), for both cocurrent and countercurrent flow configurations. In the cocurrent case the sweep gas flow (CC) has an increasing dependence on $\kappa$ and the partial pressures of the two flows meet at the maximum exchange extent, satisfying eqn (6). In the countercurrent case however both the $\mathrm{CO}_{2}$ flow and the sweep gas flow (CT) have a decreasing dependence

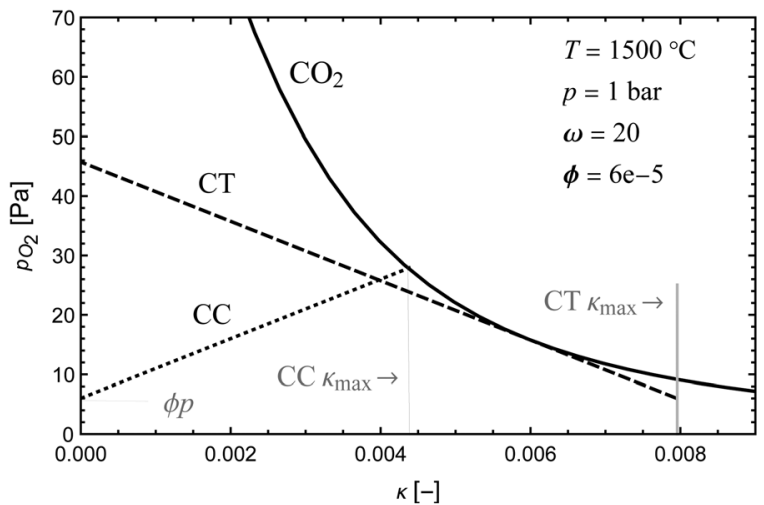

(a)

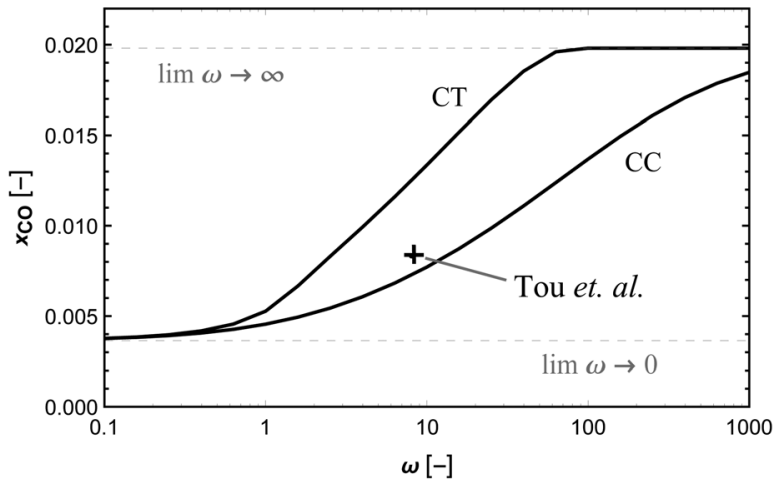

(b)

Fig. 5 (a) Plots of oxygen partial pressure vs. exchange extent $\kappa$ for the $\mathrm{CO}_{2}$ stream given by eqn (25), cocurrent sweep gas flow CC given by eqn (18), and countercurrent sweep gas flow CT given by eqn (18) with $\kappa^{\prime}=\kappa_{\max }-\kappa$. The initial oxygen partial pressure in the sweep gas $\phi p$ and the $\kappa_{\text {max }}$ in both cases are also labeled. (b) Mole fraction of $\mathrm{CO}$ in the $\mathrm{CO}_{2}$ stream plotted for cocurrent and countercurrent flow configurations at the same conditions listed in (a). Also shown is an experimental a point corresponding to Tou et al.'s experimental demonstration of this system in countercurrent configuration. ${ }^{12}$

on $\kappa$, and they share a common tangent satisfying the conditions given in eqn (8).

Fig. 5(b) shows the dependence of the mole fraction of $\mathrm{CO}$ in the product stream, on the relative flow rate $\omega$. This is equivalent to the $\mathrm{CO}_{2}$ conversion, where a value of one would indicate complete conversion. It can be seen that the countercurrent arrangement almost doubles the formation of $\mathrm{CO}$ (and $\kappa_{\max }$ ) relative to the cocurrent case for $\omega<30$. This is analogous to a countercurrent heat exchanger which can offer double the heat exchange of a cocurrent systems. In general, the conversion of $\mathrm{CO}_{2}$ to $\mathrm{CO}$ increases with increasing $\omega$, and approaches a thermodynamic limit which is determined by the oxygen impurity in the sweep gas. The sweep gas impurity was selected to match conditions reported by Tou et al., where we have also included the conversion extent measured for their countercurrent reactor. ${ }^{12}$ The experimental value lies above the cocurrent model (CC), indicating that there was a real benefit to countercurrent operation. It also lies below the countercurrent thermodynamic limit, which it should.

Using the model applied by previous authors of matching the partial pressures at both the entrance and exit of the countercurrent reactor, means that the maximum exchange 
extent $\kappa_{\max }$ only depends on the purity of the sweep gas. ${ }^{11,12}$ This is a counter intuitive result, where a fully pure sweep gas would then offer complete conversion. It can be seen by the differing shapes of the curves in Fig. 5(a), that matching both ends in the countercurrent case would not be possible, without violating the conservation of mass and/or the second law of thermodynamics (eqn (17)).

Correctly analysing the benefit of countercurrent operation for such a reactor shows that the conversion extent of $\mathrm{CO}_{2}$ (or $\mathrm{H}_{2} \mathrm{O}$ ) at $1500{ }^{\circ} \mathrm{C}$ will be very small, unless huge quantities of very pure sweep gas are fed to the reactor. The thermodynamics of this membrane reactor system for thermolysis of $\mathrm{CO}_{2}$ or $\mathrm{H}_{2} \mathrm{O}$, indicate that very high temperatures and/or very low oxygen partial pressures are required to achieve significant conversion of the reactants. This is unlikely to offer a practical or economically competitive means of converting heat to chemical energy.

\section{(b) Membrane reforming}

In the above section we used an analytical approach to solve a simple membrane countercurrent problem. In this case we look at a more complicated reaction system, and apply the methodology developed with a robust numerical analysis of the thermodynamic equilibrium. An interesting variation on the above process is to use methane instead of a sweep gas, where we then have methane partial oxidation taking place in flow 2 ,

$$
\mathrm{CH}_{4}+\frac{1}{2} \mathrm{O}_{2} \stackrel{\text { exothermic }}{\longrightarrow} 2 \mathrm{H}_{2}+\mathrm{CO} \text {. }
$$

This system is illustrated in Fig. 4(b), where $\mathrm{CO}_{2}$ and $\mathrm{CH}_{4}$ flows are separated by an oxygen selective membrane. If $\mathrm{CH}_{4}$ and $\mathrm{CO}_{2}$ are supplied in equal stoichiometry, the sum of the reactions on both sides is then dry reforming

$$
\mathrm{CH}_{4}+\mathrm{CO}_{2} \stackrel{\text { endothermic }}{\longrightarrow} 2 \mathrm{H}_{2}+2 \mathrm{CO},
$$

which consumes heat and gives two product streams, CO and $2 \mathrm{H}_{2}$ :1CO syngas. This type of membrane methane reforming has been demonstrated by several authors experimentally. ${ }^{3,16,17}$

For methane partial oxidation, there can also be the formation of $\mathrm{CO}_{2}$ and $\mathrm{H}_{2} \mathrm{O}$, and so a simplified analytical approach will not suffice. Instead the thermodynamics of the reactions was modeled using the software Cantera, ${ }^{13}$ and it's gri30 database, which contains all of the relevant species. This software uses an element potential method to equilibrate an initially defined mixture of gases by minimizing the Gibbs free energy for the system. ${ }^{18}$

Since the same reaction is taking place in flow 1 as the previous example, our exchange parameter can again be defined by eqn (14), where we do not consider (or indeed expect) the reduction of $\mathrm{CO}$ to carbon, so that $\kappa=0.5$ represents complete conversion. Here we consider the case with equal flow rates,

$$
\omega=\frac{\dot{n}_{\mathrm{CH}_{4}}}{\dot{n}_{\mathrm{CO}_{2}}}=1,
$$

at a pressure of 1 bar in both streams, and study the equilibrium limitations as a function of temperature. To apply the methodology we need to determine the oxygen chemical potential or $p_{\mathrm{O}_{2}}(\kappa)$ in both streams. For the $\mathrm{CO}_{2}$ stream this can be achieved by taking an initial mixture of $\mathrm{CO}$ and $\mathrm{O}_{2}$ corresponding to a given $\kappa$,

$$
\mathrm{CO}+\left(\frac{1}{2}-\kappa\right) \mathrm{O}_{2} \stackrel{\min \left(G_{T, p}\right)}{\longrightarrow} y_{1} \mathrm{CO}_{2}+y_{2} \mathrm{CO}+y_{3} \mathrm{O}_{2}+\ldots
$$

and finding the thermodynamic equilibrium composition at constant temperature and pressure. From the result we can determine the partial pressure of oxygen $p_{\mathrm{O}_{2}, 1}(\kappa)$. This reaction has the same equilibrium composition as the $\mathrm{CO}_{2}$ splitting reaction for a given $\kappa$ as it is simply the reverse reaction.

Similarly for the methane partial oxidation we consider an initial mixture of methane and find the thermodynamic equilibrium composition,

$$
\begin{aligned}
& \mathrm{CH}_{4}+\kappa \mathrm{O}_{2} \stackrel{\min \left(G_{T, p}\right)}{\longrightarrow} y_{1} \mathrm{CH}_{4}+y_{2} \mathrm{CO}+y_{3} \mathrm{H}_{2}+y_{3} \mathrm{H}_{2} \mathrm{O} \\
& \quad+y_{4} \mathrm{CO}_{2}+\ldots
\end{aligned}
$$

to determine $p_{\mathrm{O}_{2}, 2}(\kappa)$. Starting with a small value of $\kappa$ and incrementally increasing it, we can determine the maximum exchange by numerically checking if the condition in eqn (6) holds for the cocurrent case, and if eqn (3) holds for all $\kappa$ in the countercurrent case. This algorithm is graphically illustrated in Fig. 6, where it is important to reverse the order of the countercurrent oxygen partial pressure dependence $p_{\mathrm{O}_{2}, 2}(\kappa)$.

Fig. 7(a) shows an example of the equilibrium partial pressure profiles with respect to the exchange coordinate $\kappa$. Here it can be seen that the $\mathrm{CO}_{2}$ oxygen release profile and $\mathrm{CH}_{4}$ oxygen uptake profile in countercurrent configuration allow for complete exchange $\left(\kappa_{\max , \mathrm{CT}}=0.5\right)$ and complete $\mathrm{CO}_{2}$ conversion to $\mathrm{CO}$, even at $600{ }^{\circ} \mathrm{C}$. The cocurrent case on the other hand only exchanges about two thirds of the oxygen.

$\mathrm{CO}_{2}$ conversion alone is not enough to determine optimal operating conditions in this case. We must also consider $\mathrm{CH}_{4}$ conversion, as further oxidation of $\mathrm{CO}$ and $\mathrm{H}_{2}$ to $\mathrm{CO}_{2}$ and $\mathrm{H}_{2} \mathrm{O}$ in flow 2, can decrease the conversion of methane to syngas.

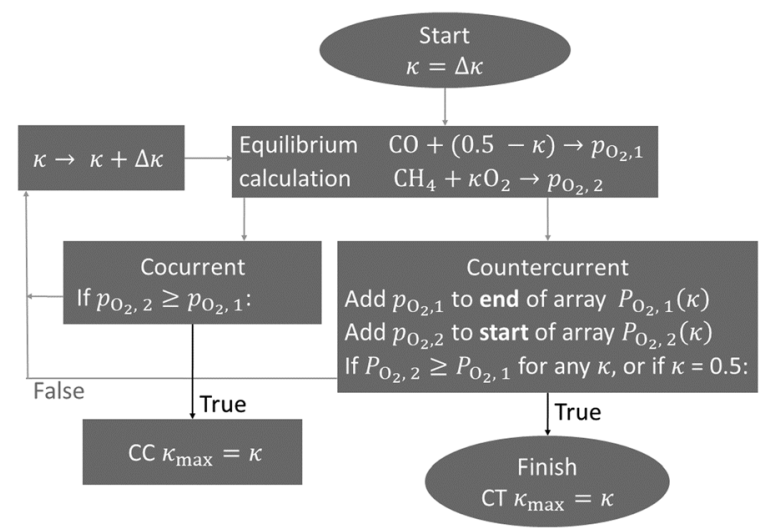

Fig. 6 The numerical algorithm used to determine the maximum possible oxygen exchange in a dry reforming membrane reactor. This was implemented in Python using Cantera, and the code has been made publicly available.† 


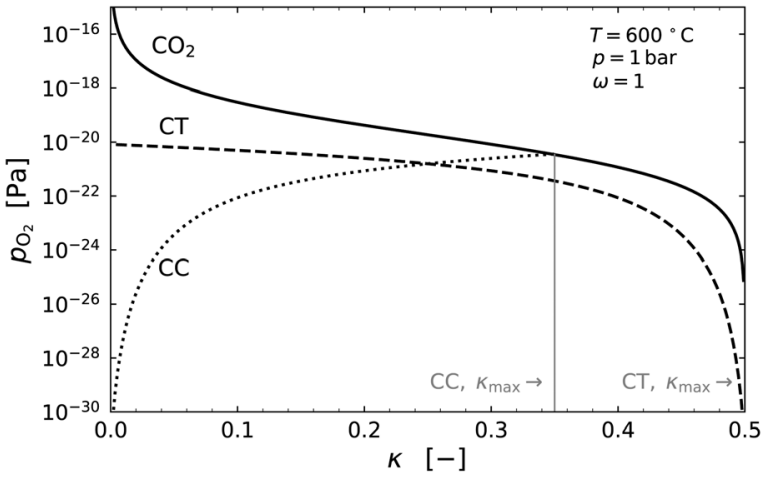

(a)

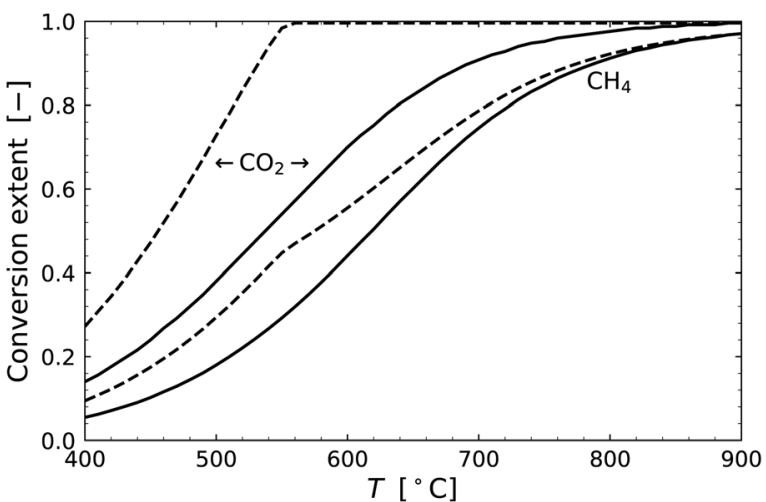

(b)

Fig. 7 (a) Plots of oxygen partial pressure vs. exchange extent $\kappa$ for the $\mathrm{CO}_{2}$ stream, the cocurrent $\mathrm{CH}_{4}$ flow $\mathrm{CC}$, and countercurrent $\mathrm{CH}_{4}$ flow $\mathrm{CT}$. (b) $\mathrm{CO}_{2}$ conversion $(=2 \kappa)$ and $\mathrm{CH}_{4}$ conversion, plotted as a function of the temperature for cocurrent with solid lines, and countercurrent with dashed lines.

The methane conversion can be determined from the mole fractions using the carbon balance,

$$
\mathrm{CH}_{4} \text { conversion }=1-\frac{x_{\mathrm{CH}_{4}}}{x_{\mathrm{CH}_{4}}+x_{\mathrm{CO}}+x_{\mathrm{CO}_{2}}},
$$

which is plotted along with the $\mathrm{CO}_{2}$ conversion in Fig. 7(b). Here it can be seen that although the countercurrent offers very promising thermodynamics for $\mathrm{CO}_{2}$ conversion at low temperatures, both countercurrent and cocurrent configurations require high temperatures of $800{ }^{\circ} \mathrm{C}$ plus, to achieve a high methane conversion and syngas production in flow 2 . The thermodynamic benefits of countercurrent are therefore mostly limited to the $\mathrm{CO}_{2}$ conversion.

In the literature the results for conversion extents by Michalsky et al., fall well short of the thermodynamic limit, ${ }^{3}$ indicating that the system may have been kinetically limited. This is supported by the work of Jin et al., who used a catalyst in their membrane reactor and achieved higher conversions. ${ }^{16}$ The reactor of Jin et al. would be best modelled as cocurrent, where the authors used a ratio of $3 \mathrm{CO}_{2}: 1 \mathrm{CH}_{4}$, i.e. $\omega=0.33$. The conversion extent trends were similar to the thermodynamic analysis presented here, with a steeper dependence on temperature, which may indicate kinetic limitations at lower temperatures. Unfortunately, in these demonstrations the reactants are diluted in inert gases for experimental analysis purposes, which makes a more quantitative comparison difficult.

\section{(c) Membrane reverse water gas shift}

It is also interesting to consider other gases than $\mathrm{CH}_{4}$ to reduce $\mathrm{CO}_{2}$ in a membrane reactor. Of particular interest is to use a hydrogen flow giving flow 2 the oxidation reaction,

$$
\mathrm{H}_{2}+\frac{1}{2} \mathrm{O}_{2} \stackrel{\text { exothermic }}{\longrightarrow} \mathrm{H}_{2} \mathrm{O} \text {. }
$$

We then have $\mathrm{CO}_{2}$ and $\mathrm{H}_{2}$ flows separated by an oxygen selective membrane. The sum of the reactions on both sides of the membrane is the reverse water gas shift (RWGS)

$$
\mathrm{H}_{2}+\mathrm{CO}_{2} \stackrel{\text { endothermic }}{\longrightarrow} \mathrm{H}_{2} \mathrm{O}+\mathrm{CO},
$$

which consumes a small amount of heat and with a membrane reactor gives two product streams, $\mathrm{CO} / \mathrm{CO}_{2}$ and $\mathrm{H}_{2} / \mathrm{H}_{2} \mathrm{O}$. This reaction is of industrial relevance for producing syngas $\left(\mathrm{H}_{2}+\mathrm{CO}\right.$ mixtures $)$ from a hydrogen source. Syngas is a highly valuable product used in many industrial processes, including gas to liquids plants for producing fuels. This is of particular interest if combined with renewable sources of hydrogen, allowing for the storage of renewable energy sources in highly valuable liquid fuels. The reverse water gas shift however typically requires very high temperatures $\left(>800{ }^{\circ} \mathrm{C}\right)$, making industrial implementation a challenge. Some concepts have been considered to reduce the temperature, such as using a steam absorbent to shift the equilibrium to higher conversion. ${ }^{19}$ The analysis here shows that using a countercurrent membrane reactor with separate $\mathrm{H}_{2}$ and $\mathrm{CO}_{2}$ streams, has promising thermodynamics for relatively low temperature operation.

Taking renewable hydrogen followed by methanol synthesis as an example application, one could produce a suitable syngas by feeding three times as much hydrogen as $\mathrm{CO}_{2}$,

$$
\omega=\frac{\dot{n}_{\mathrm{H}_{2}}}{\dot{n}_{\mathrm{CO}_{2}}}=3 \text {. }
$$

The resulting steam would then be condensed out of the hydrogen stream, both product streams would be mixed and then fed to the methanol synthesis process. The feed ratio of $3: 1$ ensures that we will have a syngas composition suitable for methanol synthesis even without complete conversion of the $\mathrm{CO}_{2}$ according to the reactions,

$$
\begin{gathered}
2 \mathrm{H}_{2}+\mathrm{CO} \rightarrow \mathrm{CH}_{3} \mathrm{OH}, \\
3 \mathrm{H}_{2}+\mathrm{CO}_{2} \rightarrow \mathrm{CH}_{3} \mathrm{OH}+\mathrm{H}_{2} \mathrm{O} .
\end{gathered}
$$

The reactor is modelled to operate at 1 bar, but since the RWGS reaction does not change the number of moles of gas, the thermodynamic conversion is independent of pressure.

Since the same reaction is taking place in flow 1 as the previous two examples, our exchange parameter is again given by eqn (14). The thermodynamic library Cantera is used to model thermodynamics of each stream. The $\mathrm{CO}_{2}$ stream was modelled according to eqn (30), to determine the oxygen partial 
pressure $p_{\mathrm{O}_{2}, 1}(\kappa)$. Similarly for the $\mathrm{H}_{2}$ stream the equilibrium is calculated according to,

$$
3 \mathrm{H}_{2}+\kappa \mathrm{O}_{2} \stackrel{T, P}{\longrightarrow} y_{1} \mathrm{H}_{2} \mathrm{O}+y_{2} \mathrm{H}_{2}+y_{3} \mathrm{O}_{2}+\ldots
$$

to determine $p_{\mathrm{O}_{2}, 2}(\kappa)$. The same numerical algorithm given shown in Fig. 6 is used to find the thermodynamic limits.

Fig. 8(a) shows an example of the partial pressure profiles with respect to the exchange coordinate $\kappa$. Here it can be seen that the $\mathrm{CO}_{2}$ oxygen release profile and $\mathrm{H}_{2}$ flow's oxygen uptake profile in countercurrent configuration allow for almost double the oxygen exchange as the cocurrent case. In the countercurrent case, more than $90 \%$ of the oxygen is exchanged corresponding to almost complete conversion of the $\mathrm{CO}_{2}$ to $\mathrm{CO}$, even at just $500{ }^{\circ} \mathrm{C}$.

The right hand side of Fig. 8 shows the conversion extent of $\mathrm{CO}_{2}$ for both cases, and for comparison an equilibrium calculation of the standard RWGS reaction with $3 \mathrm{H}_{2}+\mathrm{CO}_{2}$. As one would intuitively expect the thermodynamic conversion limit in the cocurrent case is identical to that of the standard RWGS process.

The analysis shows that a countercurrent membrane reactor has promise for a low temperature reverse water gas shift process. The author could not find any experimental work or otherwise on this particular process idea, and it may be a novelty

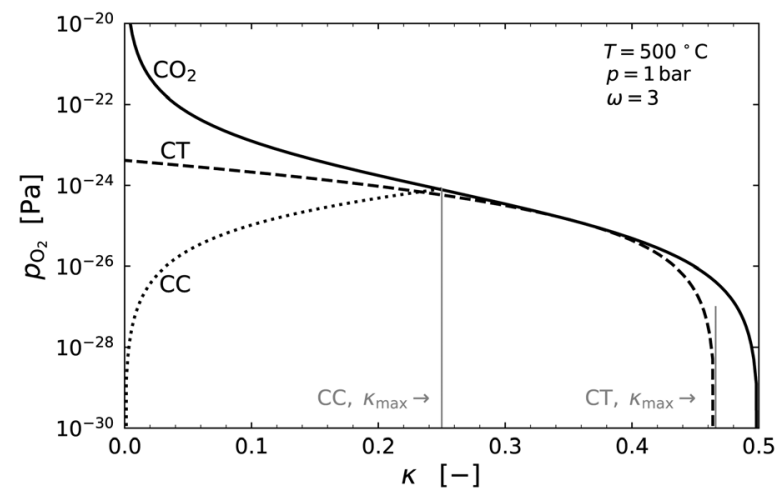

(a)

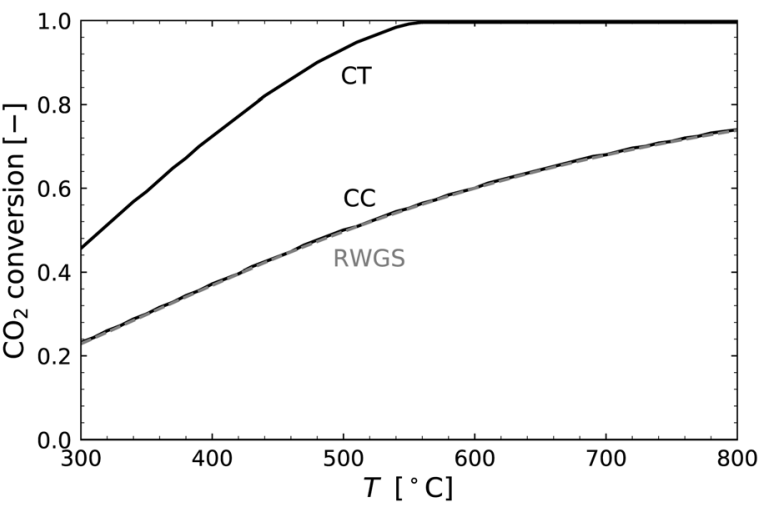

(b)

Fig. 8 (a) Plots of oxygen partial pressure vs. exchange extent $\kappa$ for the $\mathrm{CO}_{2}$ stream, the cocurrent $\mathrm{H}_{2}$ flow $\mathrm{CC}$, and countercurrent $\mathrm{H}_{2}$ flow $\mathrm{CT}$. (b) Maximum $\mathrm{CO}_{2}$ conversion $\left(=2 \kappa_{\max }\right)$ in flow 1 plotted as a function of the temperature for cocurrent and countercurrent flow configurations and for a simple co-feed reactor with $3 \mathrm{H}_{2}$ : $1 \mathrm{CO}$ (RWGS). realised in this study. A physical implementation of this system have kinetic issues at low temperatures $\left(<700{ }^{\circ} \mathrm{C}\right)$, and would likely require the use of catalysts on the membrane surfaces to realise the thermodynamic benefits illustrated in Fig. 8.

\section{(d) Ceria reduction}

Both $\mathrm{H}_{2} \mathrm{O}$ and $\mathrm{CO}_{2}$ can be split to produce fuel via a thermochemical redox cycle with ceria,

$$
\begin{gathered}
\mathrm{CeO}_{2} \stackrel{\text { endothermic }}{\longrightarrow} \mathrm{CeO}_{2-\delta}+\frac{\delta}{2} \mathrm{O}_{2} \quad T \approx 1500{ }^{\circ} \mathrm{C} \\
\mathrm{CeO}_{2-\delta}+\delta \mathrm{H}_{2} \mathrm{O} / \mathrm{CO}_{2} \stackrel{\text { exothermic }}{\longrightarrow} \mathrm{CeO}_{2}+\delta \mathrm{H}_{2} / \mathrm{CO} \quad T \approx 900{ }^{\circ} \mathrm{C}
\end{gathered}
$$

where ceria is reduced at high temperature storing chemical energy, and the reduced state is then used to split water or carbon dioxide at a lower temperature producing fuel. ${ }^{20-22}$ Ceria shows non stoichiometric behaviour, where the extent of reduction depends on both the temperature and the oxygen partial pressure $\delta\left(T, p_{\mathrm{O}_{2}}\right) \cdot{ }^{23,24}$ The reduction is the more demanding part of the cycle in terms of heat required and operation temperature. In addition it also requires a low oxygen partial pressure in order to achieve a significant reduction extent $\delta$. In practice this can be achieved using either a sweep gas or a vacuum pump.

Since the ceria is in the solid phase throughout the process, a flow of ceria particles through a reactor could be utilized, and a sweep gas could be arranged in countercurrent configuration as illustrated in Fig. 8. The oxidation step could also utilize a countercurrent reactor, but here we focus our analysis on the more critical endothermic reduction reaction.

Analysis of this system has proved to be controversial in the literature, with examples of modeling errors seen in the works of Davidson et al. ${ }^{7,8}$ They investigate this countercurrent reduction reactor with ceria, but apply the simplified assumption that partial pressures of oxygen at the exit of both streams will match the inlet of the other stream. This removes any need for a robust analysis, but predicts that oxygen partial pressures of $10^{-6}$ bar (or in principle, any pressure), can be achieved with no sweep gas at all or indeed any additional work. Despite this obviously paradoxical result, the model has been used by other authors, ${ }^{9,10}$ giving unrealistic expectations on the performance of countercurrent reactors for thermochemical redox cycles. Brendelberger et al. noticed that there was an error and published a more detailed analysis of this particular case, by assuming the partial pressure profiles share a common tangent at the ceria inlet. ${ }^{25}$ This is correct in some cases, but the profiles can also meet without a tangent at the ends, or indeed meet at any exchange extent $\kappa$. Li et al. also modeled this case, including the oxidation step of a thermochemical cycle, with a much more precise approach, combining conservation of mass with a Gibbs criterian $\mathrm{d} G_{T, p} \leq 0 .{ }^{4}$ They show very clearly that the partial pressures (or chemical potentials) in the limiting case can meet at any exchange extent, and clearly highlight the unphysical nature of previous models. The model was then utilized by these authors in a broader efficiency analysis of thermochemical fuel production. ${ }^{26}$ 
Although this particular case has already been solved, we apply the methodology formulated here, as an illustration of a system with reactions between two different phases in countercurrent flow, and indeed to compare the results to previous models.

Since oxygen is being exchanged between the two flows, we can define our exchange coordinate as

$$
\kappa=\frac{\int_{0}^{l}\left|j_{\mathrm{O}_{2}}(x)\right| \mathrm{d} x}{\dot{n}_{\mathrm{CeO}_{2}}},
$$

where $j_{\mathrm{O}_{2}}$ is the flux of oxygen from the ceria surface into the gas. Also of interest is the non-stoichiometry coefficient $\delta$ in $\mathrm{CeO}_{2-\delta}$. Assuming that the ceria starts fully oxidised $(\delta=0)$, the exchange coordinate and the ceria non-stoichiometry are related by

$$
\kappa=2 \delta,
$$

since one vacancy corresponds to half a mole of oxygen gas. Here the relative molar flow rates is an important parameter which will determine the reduction extent of the ceria,

$$
\omega=\frac{\dot{n}_{\text {sweep }}}{\dot{n}_{\mathrm{CeO}_{2}}} .
$$

The oxygen chemical potential, or oxygen activity of ceria can be converted to an equivalent oxygen partial pressure, so that we can determine $p_{\mathrm{O}_{2}, 1}(\kappa)$. This is achieved using an analytical model developed by the author, ${ }^{27}$

$$
p_{\mathrm{O}_{2}}(\delta, T)=p^{\circ}\left(\left(\frac{0.35-\delta}{\delta}\right)^{2.32} \exp \left(\frac{\Delta s_{\mathrm{th}, \mathrm{O}}}{R}\right) \exp \left(\frac{-\Delta h_{\mathrm{O}}}{R T}\right)\right)^{2} .
$$

with the change in enthalpy per mole of atomic oxygen $\Delta h_{\mathrm{O}}=$ $430 \mathrm{~kJ} \mathrm{~mol}^{-1}$ and the change in thermal entropy per mole of atomic oxygen $\Delta s_{\mathrm{th}, \mathrm{O}}=165 \mathrm{~kJ} \mathrm{~mol}^{-1} \mathrm{~K}^{-1}$. This model matches very well with experimental data in the literature at the temperatures of interest. ${ }^{23,28}$ Subbing in $\delta=\kappa / 2$ gives the desired equation for the ceria stream.

For the sweep gas $p_{\mathrm{O}_{2}, 2}(\kappa)$ is given again by eqn (18), where the substitution $\kappa^{\prime}=\kappa_{\max }-\kappa$ is used for the countercurrent case. This gives all the necessary formulae to use the methodology, according to the conditions given in eqn (6)-(8).

Fig. 9(a) shows an example of the limiting case, where the temperature $T$ and oxygen impurity in the sweep gas $\phi$, were selected to match the experimental demonstration of a countercurrent reactor performed by Scheffe et al. ${ }^{29}$ They experimentally demonstrated ceria reduction with a stream of falling particles (average size $12 \mu \mathrm{m}$ ) and a countercurrent sweep gas removing the oxygen, where I have used the data from Fig. 9 in their work for comparison. In Fig. 9(b), it can be seen that countercurrent operation offers an improvement in species transfer, relative to the cocurrent case, by a factor of approximately 1.5 for the conditions considered and for $\omega<50$. It can also be seen that the experimental values measured by Scheffe are greater than the thermodynamic limit for a cocurrent reactor, but less than he limit for a countercurrent reactor,

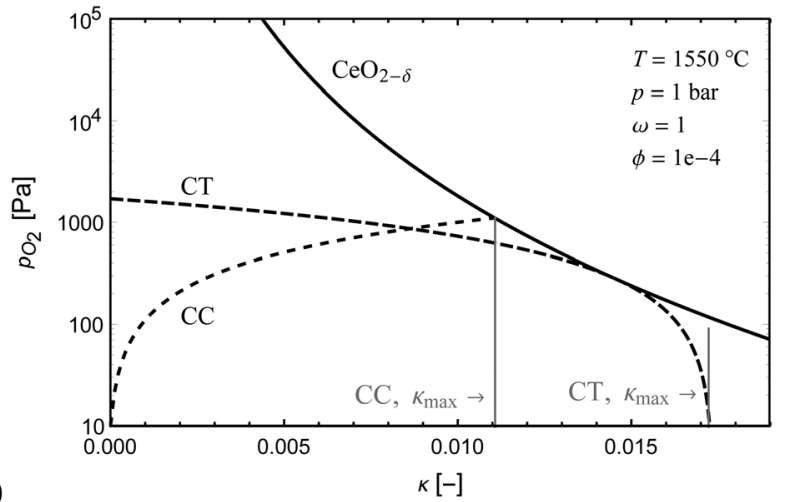

(a)

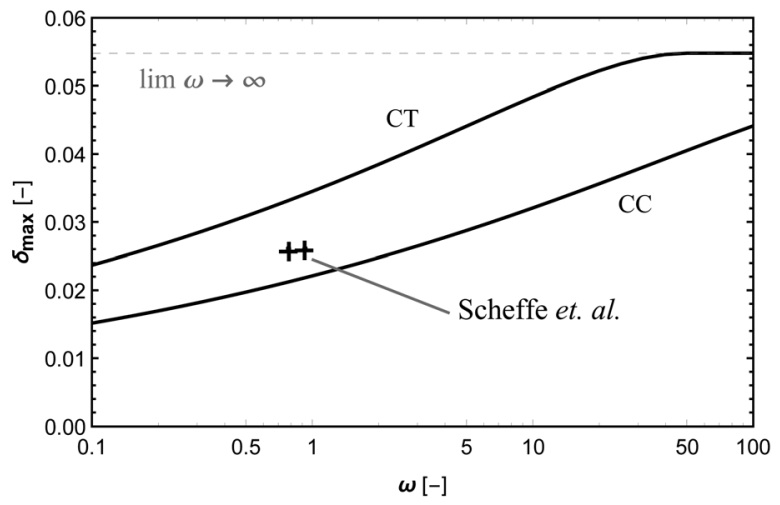

Fig. 9 (a) Plots of oxygen partial pressure vs. exchange extent $\kappa$ for the flow of $\mathrm{CeO}_{2}$ particles, and the sweep gas in cocurrent flow $\mathrm{CC}$, and countercurrent flow CT with $\kappa^{\prime}=\kappa_{\max }-\kappa$. (b) Maximum reduction extent $\delta_{\text {max }}$ plotted against the relative flow rate $\omega$, for the conditions listed in (a). Also indicated on the plot are the $\omega \rightarrow \infty$ limit, and the experimental results of Scheffe et al. for a countercurrent reactor.

and so support the model. The lower non-stoichiometry in the experiment could be due to the very short residents time for the particles in the hot zone of just one second, or partial reoxidation of the material after exiting the hot zone. The thermodynamic model is also very idealised and does not consider diffusion of oxygen species along the direction of flow, which could also play an important role at such high temperatures. Nevertheless, it has served it's function well, which is to set an upper bound on the species transfer.

From the results it can also be seen that huge quantities of sweep gas are required in both cases to produce a very small non-stoichiometry in the ceria, which is well supported experimentally for both cocurrent and countercurrent reactors. ${ }^{21,29}$ This is in stark contrast to the results obtained using a simplified model. ${ }^{8}$ The model results do however, agree with the work of Li et al..$^{4,25}$

\section{Conclusions}

The methodology presented should be very valuable in determining the limits of countercurrent reactor systems. Here we apply it to a number of cases in thermochemical fuel production, and the results indicate that countercurrent systems can offer higher potential for species transfer, typically on the order 
of 1-2 times greater than that of a cocurrent reactor in the cases analysed. This will depend on the specific reactions in the streams and values deviating from this general rule of thumb could be possible. Nevertheless this is a good first guess in most cases, and is analogous to the idea that a countercurrent heat exchanger can double the heat transfer relative to a cocurrent one. The results of the model are also supported by some experimental demonstrations of countercurrent reactors, where species exchange extents were greater than the cocurrent model, but less than our upper limit for countercurrent. Finally the analysis also highlights that an oxygen permeable membrane reactor has promising thermodynamics for low temperature reverse water gas shift using a countercurrent configuration.

\section{Conflicts of interest}

There are no conflicts of interest to declare.

\section{Acknowledgements}

Many thanks to Professor Aldo Steinfeld for his encouragement and support to pursue this manuscript as an independent research work, and to Maria Tou for many informative discussions on membrane reactors.

\section{References}

1 J. Piiper and D. Schumann, Respir. Physiol., 1967, 2, 135-148.

2 Y. Shah, B. G. Kelkar, S. Godbole and W.-D. Deckwer, AIChE J., 1982, 28, 353-379.

3 R. Michalsky, D. Neuhaus and A. Steinfeld, Energy Technol., 2015, 3, 784-789.

4 S. Li, V. M. Wheeler, P. B. Kreider and W. Lipiński, Energy Fuels, 2018, 32, 10838-10847.

5 Y. Lee, Z. Wu and R. Torget, Bioresour. Technol., 2000, 71, 29-39.

6 D. R. Parisi and M. A. Laborde, Chem. Eng. J., 2004, 104, 35-43.

7 R. Bader, L. J. Venstrom, J. H. Davidson and W. Lipiński, Energy Fuels, 2013, 27, 5533-5544.

8 P. T. Krenzke and J. H. Davidson, Energy Fuels, 2015, 29, 1045-1054.

9 L. J. Venstrom, R. M. De Smith, R. Bala Chandran, D. B. Boman, P. T. Krenzke and J. H. Davidson, Energy Fuels, 2015, 29, 8168-8177.
10 B. D. Ehrhart, C. L. Muhich, I. Al-Shankiti and A. W. Weimer, Int. J. Hydrogen Energy, 2016, 41, 19904-19914.

11 L. Zhu, Y. Lu and S. Shen, Energy, 2016, 104, 53-63.

12 M. Tou, R. Michalsky and A. Steinfeld, Joule, 2017, 1, 146-154.

13 D. Goodwin, H. Moffat and R. Speth, Cantera: An ObjectOriented Software Toolkit for Chemical Kinetics, Thermodynamics, and Transport Processes, Version 2.3.0, 2017, DOI: 10.5281/zenodo.170284.

14 E. A. Fletcher and R. L. Moen, Science, 1977, 197, 1050-1056.

15 J. E. Noring, R. B. Diver and E. A. Fletcher, Energy, 1981, 6, 109-121.

16 W. Jin, C. Zhang, X. Chang, Y. Fan, W. Xing and N. Xu, Environ. Sci. Technol., 2008, 42, 3064-3068.

17 R. V. Franca, A. Thursfield and I. S. Metcalfe, J. Membr. Sci., 2012, 389, 173-181.

18 W. R. Smith, Theory and Algorithms, 1982.

19 B. Carvill, J. Hufton, M. Anand and S. Sircar, AIChE J., 1996, 42, 2765-2772.

20 B. Bulfin, J. Vieten, C. Agrafiotis, M. Roeb and C. Sattler, J. Mater. Chem. A, 2017, 5, 18951-18966.

21 W. C. Chueh, C. Falter, M. Abbott, D. Scipio, P. Furler, S. M. Haile and A. Steinfeld, Science, 2010, 330, 1797-1801.

22 D. Marxer, P. Furler, M. Takacs and A. Steinfeld, Energy Environ. Sci., 2017, 10, 1142-1149.

23 R. Panlener, R. Blumenthal and J. Garnier, J. Phys. Chem. Solids, 1975, 36, 1213-1222.

24 B. Bulfin, A. J. Lowe, K. A. Keogh, B. E. Murphy, O. Lubben, S. A. Krasnikov and I. V. Shvets, J. Phys. Chem. C, 2013, 117, 24129-24137.

25 S. Brendelberger, M. Roeb, M. Lange and C. Sattler, Sol. Energy, 2015, 122, 1011-1022.

26 S. Li, V. M. Wheeler, P. B. Kreider, R. Bader and W. Lipiński, Energy Fuels, 2018, 32, 10848-10863.

27 B. Bulfin, L. Hoffmann, L. de Oliveira, N. Knoblauch, F. Call, M. Roeb, C. Sattler and M. Schmuecker, Phys. Chem. Chem. Phys., 2016, 18, 23147-23154.

28 M. Takacs, J. Scheffe and A. Steinfeld, Phys. Chem. Chem. Phys., 2015, 17, 7813-7822.

29 J. R. Scheffe, M. Welte and A. Steinfeld, Ind. Eng. Chem. Res., 2014, 53, 2175-2182.

30 S. Li, P. B. Kreider, V. M. Wheeler and W. Lipiński, J. Sol. Energy Eng., 2019, 141, 021012. 